\title{
Investigating the Impact of Culture on Evacuation Behavior - A Turkish Data-Set
}

\author{
EDWIN R. GALEA, MARKUS SAUTER, STEVEN J. DEERE, and LAZAROS FILIPPIDIS \\ Fire Safety Engineering Group \\ University of Greenwich \\ London SE10 9LS UK
}

\begin{abstract}
In this paper results from an unannounced evacuation trial conducted within a library in Izmir Turkey are presented and discussed. This experimental evacuation is part of a large international study investigating the impact of culture on evacuation behavior. In addition, a framework to enable the systematic analysis of Response Phase behaviors is presented and applied to the trial data. The framework not only provides a consistent method for describing Response Phase behavior, but also provides a framework for classifying and quantifying the Response Phase other than simply using the overall response time. An empirical response time model, based on data generated using the framework is also presented and applied to the evacuation trial data. The empirical response time model produces a prediction for the average response time for the trial population which is within $11 \%$ of the measured value. In addition to presenting Response Phase data, a data-set suitable for the validation of evacuation models is also presented. Computer simulations which attempt to reproduce the trial are compared to the validation data-set. While initial results are promising, analysis suggests that a model representation of exiting behavior through the exit turnstile is necessary.
\end{abstract}

KEYWORDS: evacuation, response time, validation, culture.

\section{INTRODUCTION}

Project BeSeCu (Behavior, Security and Culture) [1] is an EU FP7 funded research project with the aim of studying how people react in an emergency, and to determine whether there are any cultural factors - both social culture and fire safety culture - which impact emergency behavior. By social culture we mean a society's shared system of values, beliefs and attitudes that influences that society's perception of the world and its behavioral response to both every day encounters and unusual incidents. When discussing social cultures it must be emphasized that we are not talking about individuals but of large populations and so are attempting to compare one normal distribution of behavioral response for one population with that of another. Aspects of social culture which may differ from one society to another include degree of physical contact, nature and extent of eye contact, degree of physical social separation, sense of community, feelings of fatalism, levels of machismo, risk perception, response to authority, acceptable levels of public and private signs of emotion, etc. By fire safety culture we mean the regulatory fire safety framework, supported by legislation, prescriptive guidance, education efforts, performance guides and most important enforcement that exists within a society. This top-down safety culture (where safety levels are imposed) is, to some degree, supported by a bottom-up expectation of the public regarding safety levels in general. This extends far beyond fire safety with the public having high expectations regarding the functionality and safety levels reached in all aspects of life.

It is often suggested that differences in culture may influence the response of the public to emergency procedures. Specifically, it is hypothesized that the differences in both the social and fire cultures may result in differences in response behaviors and response times. As part of project $\mathrm{BeSeCu}$, evacuation behavior is being investigated through surveys of people who have had first-hand experience of real emergencies and through a series of unannounced evacuation trials. The surveys are being conducted in several countries - namely, the Czech Republic, Germany, Italy, Poland, Spain, Sweden, Turkey, and the UK. With samples including adults of different ages, gender, socio-economic status, and ethnic groups, this part of the project is expected to generate an evidence base of inter-individual differences that may be used to enhance communications and procedures in emergency interventions. In order to have a more rounded perspective of communications and procedures, the vast professional experiences of first responders from different regions and ranks are also being collected and analyzed. The experimental component of project $\mathrm{BeSeCu}$ addresses issues associated with Response Phase behaviors and the impact of culture. The 
evacuation process can be considered to comprise two broad phases, called the Response Phase and Evacuation Movement Phase [1,2]. It is now widely accepted that a key factor which can determine the success of an evacuation is the speed with which occupants respond to the call to evacuate and begin purposeful movement to a place of safety, commonly referred to as the response time [2-6]. In practical fire engineering applications it is common practice to assume that evacuation related data can be reliably applied more widely than the context from which the data was collected. In particular, this type of data is often applied to situations within cultural environments - both social and fire - different to that from which the data was collected. The majority of readily available published evacuation data (both response time and total evacuation time) that is used in fire engineering applications throughout the world originates from a small number of countries with broadly similar cultural backgrounds; i.e., UK, USA, Canada, Australia and New Zealand. There has been very little effort in exploring the impact of culture on fire evacuation behavior, especially outside of the small number of countries mentioned. Here we take culture to mean both the social culture and the fire safety culture of the society that the occupants belong to. This begs the question, can response time data collected from predominately Western European/North American type social cultures (UK, Australia, Canada, USA, New Zealand) be applied in significantly different social cultures such as in Japan, China, Korea, Turkey, Czech Republic, Brazil, etc? Is there a cultural component to the time required to complete the Response Phase? Furthermore, can response time data derived from countries with a well developed fire culture and legislative framework e.g., UK, Australia, Canada, USA, New Zealand, Japan be applied in countries with a less well developed fire culture and legislative framework e.g. Turkey, Brazil, Korea, Czech Republic, etc? Does a well developed fire culture influence the time required to complete the Response Phase and commence the Evacuation Movement Phase?

The experimental component of project $\mathrm{BeSeCu}$ involves three unannounced library evacuations run in the Czech Republic, Turkey and Poland. Response Phase behaviors derived from these trials will be compared with data generated from a similar evacuation conducted in the UK. These comparative studies are based not simply on response times but on a framework, developed as part of project $\mathrm{BeSeCu}$, to describe Response Phase behaviors [2]. In an earlier paper the evacuation behavior framework was presented along with a response time data-set derived from the first evacuation experiment conducted in the Czech Republic [1]. In this paper we present the evacuation data-set derived from the second evacuation trial conducted in Turkey. This data-set also consists of a full validation data-set which includes egress times. Furthermore, the predictive response time model developed in [1] is applied to the Turkish data-set to evaluate its robustness.

\section{RESPONSE PHASE BEHAVIORAL FRAMEWORK}

Within the proposed evacuation behavior framework [2], the evacuation process is considered to comprise of two broad phases the; Response Phase and Evacuation Movement Phase (see Fig. 1). The framework attempts to convey the nature of the human factors processes that characterize Response Phase behaviors. This description not only provides a consistent method for describing Response Phase behavior, but also provides a useful framework for classifying and quantifying the Response Phase other than simply using the overall response time. Using this framework we may eventually be able to develop predictive models to estimate response times. A key part of the Response Phase analysis is the determination of the Notification, Cognition and Activity stage start and end points [2] (see Fig. 1).

\section{Notification Stage}

The first stage of the Response Phase is the Notification stage (see Fig. 1). In the Notification stage, notification cues such as an alarm conveys to the occupants that an unusual and potentially hazardous event has occurred, requiring the occupants to evacuate. The notification cues may be a traditional alarm such as a siren or an alarm bell, a voice alarm, intervention by staff or environment cues such as the smell of smoke etc. The start of the Notification stage is marked by the occupants being exposed to the first cues. During the Notification stage the occupant may (knowingly or unknowingly) ignore the cues and continue with their normal activities as they have not accepted or recognized that the cues are relevant to their situation. For each exposed occupant, the end of the Notification stage is marked by the occupants responding to the notification cues by mentally and/or physical disengaging from the tasks they were previously involved in and the recognition that the cue(s) they have been subjected to indicate that something unusual may be occurring in their environment. At the end of the Notification stage, while the occupants are alerted that 
something unusual is occurring in their environment, they have not started to physically react to the situation. The end of the Notification stage marks the beginning of the Cognition stage.

\section{Cognition Stage}

During the Cognition stage the alerted occupants interpret the information provided by the initial notification cues, and potentially other sources of information (e.g. further incident related cues, staff intervention, etc), and decide how they should respond (see Fig.1). There are essentially three broad types of response the occupant may decide to undertake:

- In the first type of response, the initial notification cues have been insufficient to convey to the occupant the immediate need for evacuation and so the occupant re-engages in their previous activity until further cues or information is received. In this case, the Cognition stage continues until one of the other two possible responses occur.

- In the second type of response, the occupant has recognized the initial notification cues and as a result, immediately commences evacuation movement without undertaking any other activity. Alternatively, the occupant may have ignored the initial notification cues and re-engaged in their pre-notification activity but then acknowledges additional cues and as a result immediately commences evacuation movement without undertaking any other activity. In these cases the end of the Cognition stage also marks the end of the Response Phase and the beginning of the Evacuation Movement Phase.

- In the third type of response, the occupant acknowledges that the notification cues indicate that something potentially hazardous is occurring in their environment and as a result undertakes a series of Action and/or Information tasks, marking the start of the Activity stage. In this case, cognitions may be occurring at the same time as activities, activities could be sparking new cognitions and cognitions could initiate one or more new activities. As a result, the Activity stage can run in parallel to the Cognition stage. However, the physical duration of the Cognition stage is less well defined as it may have several possible end points and so is taken to run to the end of the Activity stage.

\section{Activity Stage}

At the start of the Activity stage the occupant performs a series of Information and/or Action tasks $[1,7]$ which were conceived during the Cognition stage (see Fig. 1). These are defined as follows:

- An Action task involves the occupant physically undertaking an activity such as: shutting down a work station; packing work items; packing/collecting personal belongings in the immediate vicinity; physically moving to another location to perform an action (e.g. fight fire, collect an item).

- An Information task involves the occupant seeking, providing or exchanging information concerning the incident or required course of action and may include; calling someone on the phone to seek / provide information; seeking / providing information in person; engaging with electronic media (e.g. television, radio, text services, etc); investigating the incident. Information tasks may thus involve the physical movement of the occupant; however, what distinguishes this activity from an Action task is the end purpose of the movement i.e. to obtain information or to undertake a physical task.

It is hypothesized that the Cognition stage may run in parallel to the Activity stage, and so the occupant may return to the Cognition stage to interpret new information gained, assess their current situation and contemplate their next course of action, which may in turn require further Action/Information tasks to be executed. Thus, during the Activity stage, there may be brief periods where the occupant appears to be undertaking no specific task. The start of the Activity stage is marked by the commencement of the planned tasks while the end of the Activity stage is marked by the completion of all tasks conceived during the Cognition stage. The end of the Activity stage usually denotes the end of the Response Phase and the beginning of the Evacuation Movement Phase. It is only at the start of the Evacuation Movement Phase that the occupant begins their purposeful movement to an exit or stair or place of safety. 


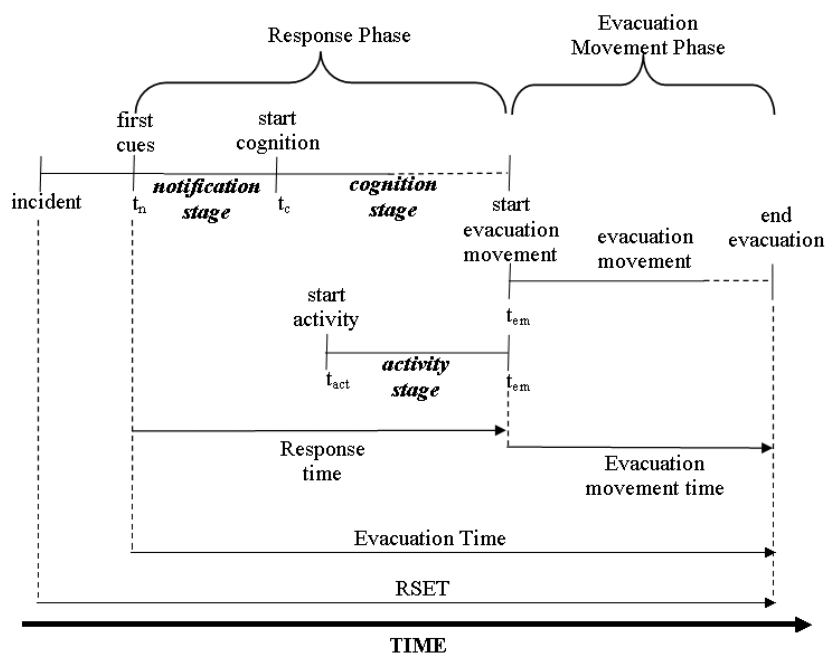

Fig. 1. Framework for describing evacuation behavior.

\section{VIDEO ANALYSIS AND EQUIPMENT SET UP}

Video footage collected from the evacuation trials is analyzed frame by frame to determine response times and Response Phase behaviors. In this type of analysis, for the most part, it is not possible to determine the end of the Notification and start of the Cognition stages. This is because from the video footage alone it is difficult to determine when incident related cognitions occur. However, using the video footage it is possible to determine when someone's behavior changes from that which they were involved in prior to the Notification stage to those behaviors defined in the Activity stage such as seeking information, packing away belongings and shutting down equipment, etc. Thus, in the video analysis, the Cognition and Activity stages are combined and referred to simply as the Activity stage. As a result the video analysis provides the following information:

- The start of the Response Phase (i.e. the start of the Notification stage) which is indicated by the first notification cues e.g. the sound of the alarm or staff intervention.

- The end/start of the Notification/Activity stage where people begin to perform action and information tasks prior to starting the Evacuation Movement Phase.

- The number, type and duration of Action and Information tasks performed.

- The end point of the Activity stage and the commencement of the Evacuation Movement Phase.

- Response Time.

Evacuation behavior was captured using 29 digital video cameras and a questionnaire administered to the evacuees post-evacuation. The questionnaire consisted of 29 questions and was translated into Turkish, the native language of the trial participants. The trial took place on the 2 March 2010 in the Izmir Yuksek Teknoloji Enstitusu university library.

\section{THE EVACUATION TRIAL}

The library has two floors and students have access to two stairs and two routes out of the library, the main entrance, with two doors, and an emergency exit which is normally locked and not in normal use. To exit the building via the main exit, occupants must first pass through a turnstile. In normal usage, students must pass their ID cards over a card reader while during emergency situations the turnstile is set to free-swing. The library building has mixed occupancy and accommodates staff offices, computer rooms, study areas, reading rooms and a canteen. The library has 19 members of staff and an average daily usage of 800-900 visitors. At any one time there may be between 50 and 300 visitors within the library. The alarm system within the library consists of a combination of tones and a recorded voice message which are run concurrently. Apart from the evacuation experiment conducted for this project, there had never been a fire drill in the building. Furthermore, the student population had never used the emergency exit as it is normally locked. To the best knowledge of the library staff, the fire alarm system had never been tested and 
a recorded message had not been set by the staff. It is worth noting that all of these issues (e.g. locked emergency exit, lack of drills, etc) are examples of how the local fire culture can impact overall evacuation performance. An alarm message was recorded which requested the immediate evacuation of the library. The $7.5 \mathrm{~s}$ duration message was repeated continuously throughout the evacuation. From the questionnaires that were completed, at the time of the alarm, there were 74 students within the library, 43 on the ground floor and 15 on the first floor. According to the video analysis, there were 120 people in the library, including library staff, cleaners and students. In total there were 106 students within the library, 56 on the ground floor, 30 on the first floor and 20 students for which their initial location is unknown. Of the 106 students, it was possible to determine response time and response behaviors for 51 students, 35 on the ground floor and 16 on the first floor.

\section{TRIAL RESULTS AND DISCUSSION}

The presentation and discussion of the experimental results is in three parts, first the Response Phase data are presented, then the predictive response time model is investigated using the data from this experiment and finally the exit time data is presented.

\section{Analysis of Response Phase Data}

A total of 111 questionnaires were given out and 87 were returned and of these 74 respondents were identified as students. From the questionnaire, 54\% (40) of the student population was male, 46\% (34) were female and $97 \%$ (72) were in the age range 18-24 and 3\% (2) were in the age range 25-34. From the video analysis, the Response Phase behavior of 51 students could be analyzed, of which 25 were males and 26 were females. Unlike in the Czech Republic evacuation experiment [1], there was no staff interaction with the students during the Response Phase and so the students only reacted to the alarm. As a result there is only one group of response behaviors to be considered. The response time is determined from the start of the alarm (first cue and start of the Notification stage), to the point where the person began purposive movement towards an exit (end of Activity stage and start of Evacuation Movement Phase). The response time distribution for the population is presented in Fig. 2. As can be seen from Fig. 2, the response time distribution approximately follows a log-normal profile, with $49 \%$ of the population responding within 40 $\mathrm{s}, 90 \%$ of the population responding within $100 \mathrm{~s}$ and $92 \%$ of the population responding within $150 \mathrm{~s}$. The average response time extracted from the 51 analyzed people was $56.1 \mathrm{~s}$, and ranged from $11 \mathrm{~s}$ to $181 \mathrm{~s}$. The log-normal curve shown in Fig. 2 has a $\log$ mean of 3.78 and a log standard deviation of 0.72.

Simply considering the overall response time does not provide insight into the nature of the Response Phase behaviors. To gain a better understanding of the factors contributing to the observed response time distributions it is necessary to decompose the Response Phase into the constituent stages as identified in Fig. 1. This is achieved through detailed analysis of the video footage. If we consider the duration of the Notification stage we note that $61.2 \%$ (30 people) of the population have completed the Notification stage and disengaged from their pre-notification activities within $5 \mathrm{~s}$ of the start of the notification cues. The longest Notification stage was $56.2 \mathrm{~s}$, achieved by $2 \%$ ( 1 person) of the population while the average duration of the Notification stage is $10.0 \mathrm{~s}$. The nature of the personal behaviors exhibited during the Activity stage can also be analyzed in detail. A total of 429 Action and Information tasks were identified during the Response Phase for the entire building population, 174 individual Action tasks (AT) and 255 Information tasks (IT). The average number of ATs undertaken is 3.5 while the average number of ITs is 5.1. On average a person undertakes more ITs than ATs and undertakes a total of 8.6 tasks prior to commencing the Evacuation Movement Phase. Furthermore, the distribution of the Action, Information and total number of tasks undertaken by the entire building population each follow an approximate log-normal distribution. 


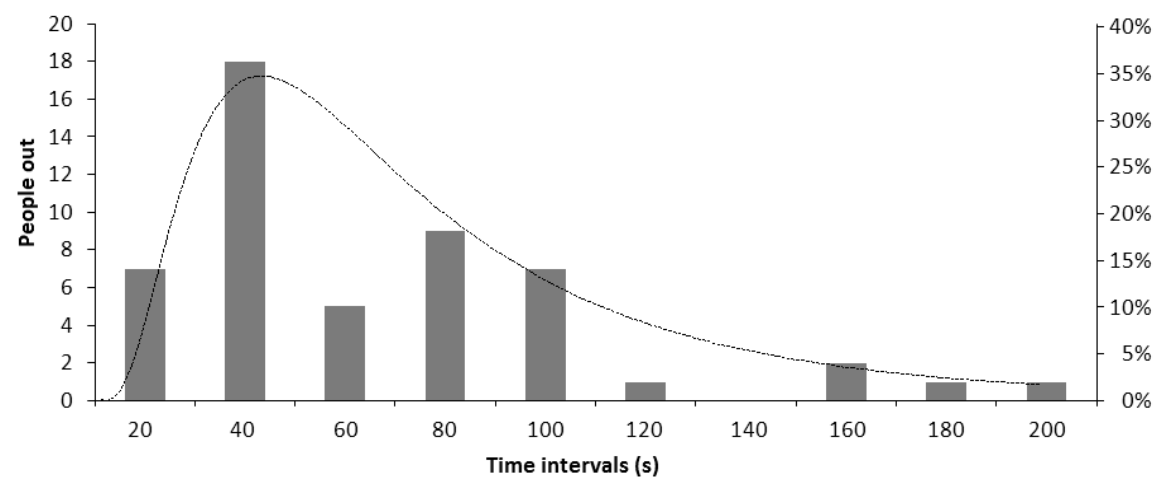

Fig. 2. Response time distribution for student population.

The most common combination of tasks was 3 ATs and 3 ITs and this combination was completed by 4 people. The most common number of ATs was 1 and 4 performed by 10 people and the most common number of ITs was 3 performed by 10 people. The maximum number of tasks performed by a single person was 23 tasks (made from a combination of ATs and ITs), while the maximum number of ATs performed by a single person was 13 and the maximum number of ITs performed by a single person was 12 . In addition, the most common ITs, expressed as a percentage of the total number of ITs were; "Acquire information from the environment (looking, listening)" 63\%, and "Engage in information exchange" 27\%. The most common ATs, expressed as a percentage of the total number of ATs were "Collect belongings" 29\%, "Pick up bag" 24\%, "Shut down computer" 9\% and "Work at computer" 6\%. The time to undertake each individual task can be determined from the video footage. However, it is difficult to reliably measure short duration activities when there is uncertainty in the start and end points of the activity. To increase the reliability of the measurement, the duration of only multiple tasks of the same type were measured. Thus, measurements were made for sequences of tasks in which at least two tasks of the same type were undertaken consecutively and which followed on immediately from the previous task. Using this approach the duration of 92 ATs and 170 ITs were measured. The average duration of a single AT was determined to be $5.2 \mathrm{~s}$ while the average duration of an IT was determined to be $4.7 \mathrm{~s}$. Thus, on average, for this population ATs take approximately $10 \%$ longer than ITs. A similar analysis of Response Phase behaviors has already been undertaken for an evacuation of a library in the Czech Republic. Two further analyses are currently underway for two other library evacuations, one conducted in Poland and one in the UK. When these are completed all four data-sets will be compared in an attempt to identify potential similarities and differences which may be attributable to cultural differences in the populations.

\section{Predictive Response Time Model}

An empirical model was developed to predict the average response time for a population based on the Response Phase parameters defined using the framework (see Eq. 1) [1].

$$
\begin{gathered}
R T=[N T+(N A T \times A T T)+(N I T \times I T T)]-\left[W \times X \times F O L_{\max } \times A T T\right] \\
+[Y \times Z \times T N T \times(A T T+I T T) / 2]
\end{gathered}
$$

Where: $R T=$ Response Time $(\mathrm{s}) ; N T=$ Notification Time $(\mathrm{s}) ; N A T=$ Number of Action Tasks; NIT = Number of Information Tasks; $T N T=$ Total number of tasks; $I T T=$ Information Task time (s); $A T T=$ Action Task time $(\mathrm{s}) ; F O L_{\max }=$ Max number of overlapping tasks; $W, X, Y$ and $Z$ are constants.

In this expression the first three terms on the right side measure the sequential nature of the Notification and Activity stages. The bulk of the time in the Activity stage is simply made up of the time required to perform a number of ATs and ITs. The duration of the Notification stage, the number and type of tasks and the average duration of an AT and IT are all dependent on the nature of the notification process, e.g. staff intervention and the type of environment e.g. library. It is also possible that there is a cultural component to each of these parameters. The fourth term is a correction factor that takes into consideration that a 
proportion of ATs can be performed simultaneously with ITs thereby reducing the calculated response time. The maximum number of overlapping tasks is dependent on the relative number of ATs and ITs and is given by:

$$
\begin{aligned}
& F O L_{\max }=N I T \text { if } N A T \geq N I T \\
& F O L_{\text {max }}=N A T \text { if } N A T<N I T
\end{aligned}
$$

In Eq. $1, \mathrm{~W}$ is a measure of the likelihood that tasks overlap and $X$ is a measure of the degree of task overlap. The last term in Eq. 1 is a correction factor that takes into account brief periods during the Activity stage where no task is undertaken. This is assumed to be when the occupant is considering their next course of action and so represents a brief Cognitive stage or cognition pause. In this case the Cognitive stage does not occur in parallel to the Activity stage but in sequence to it. To compensate for this a small portion of time, representing the cognition pause, is added to the total response time. Cognition pauses can occur following each of the TNT tasks. However, they are observed to occur significantly less frequently then this, and the proportion of tasks that are followed by a cognition pause is represented by a constant $Y$. In most cases the cognition pause is a very brief period of time, while in some cases it can be relatively long. It is assumed to be a multiple $Z$ of the average time for ATs and ITs. The constants $W, X, Y$ and $Z$ in Eq. 1 were determined from analysis of the video footage derived from the Czech Republic evacuation trial. From that analysis, the constants $W, X, Y$ and $Z$ were estimated to be $0.25,1.0,0.25,0.2$ [1]. The same values for the constants will be used in this analysis. It should be noted that the constants $W, X, Y$ and $Z$ may be dependent on the nature of the notification process; e.g. voice alarm and the type of environment e.g. library. It is also noted that it may be possible that there is a cultural component to each of these constants. In this assessment, we will investigate if the constant values derived from one library evacuation can be applied to an evacuation in a different library. Using the assumed values for these constants, Eq. 1 simplifies to:

$$
\begin{gathered}
R T=[N T+(N A T \times A T T)+(N I T \times I T T)]-\left[0.25 \times F O L_{\max } \times A T T\right] \\
+[0.05 \times T N T \times(A T T+I T T) / 2]
\end{gathered}
$$

Using Eq. 3, it is possible to estimate the response time for the population. Based on the data derived from the Turkish evacuation trial, we have the following average values for each parameter,

$N T=9.98 \mathrm{~s}, N A T=3.48, N I T=5.10, T N T=8.58, A T T=5.18 \mathrm{~s}, I T T=4.74 \mathrm{~s}, F O L_{\max }=N A T$

Using the above values in Eq. 3 the average response time is $R T=49.80 \mathrm{~s}$, whereas the measured average response time for the population was $56.14 \mathrm{~s}$. Thus using the empirical response time model, the average response time is under-predicted by $11.3 \%$. As can be seen, the empirical response time model provides a good estimation of the average response time for the population. From the earlier analysis of the evacuation trial in the Czech Republic, the empirical response time model produced average response times which were within $1.6 \%$ of the measured average response time for that population [1]. Thus the constants used in the empirical response time model appear to be reasonably robust, providing a good level of agreement for two evacuation trials conducted in two different libraries in two different countries. Data from two additional evacuation trials, one in Poland and one in the UK is currently being analyzed. Once this analysis is complete, the empirical response time model will be applied to these data-sets and its level of agreement with the measured data will be established. If the empirical response time model can be demonstrated to be robust, its value goes beyond simply being a means of determining average response times for a population. Of more importance is the insight it provides into the behavioral factors driving the response time. Using this approach it will be possible to estimate the impact of introducing technical or procedural measures to address various behavioral determinants of response time such as the duration of the average IT, or the number of ITs that are performed by a population.

\section{Exit Time Data}

One of the aims of the $\mathrm{BeSeCu}$ project was to collect evacuation data that could be used as validation datasets for evacuation models. Here we present exit time data and initial conditions for the Turkish Evacuation Validation Data-set (TEVDS). As stated previously there were 120 people within the library, 14 staff, including library staff and cleaners and 106 students. This number was determined from the analysis of the 
video surveillance of each of the two exits. Presented in Fig. 3. are the exit curves for each of the two exits. These graphs show the exit time for all the occupants of the library.

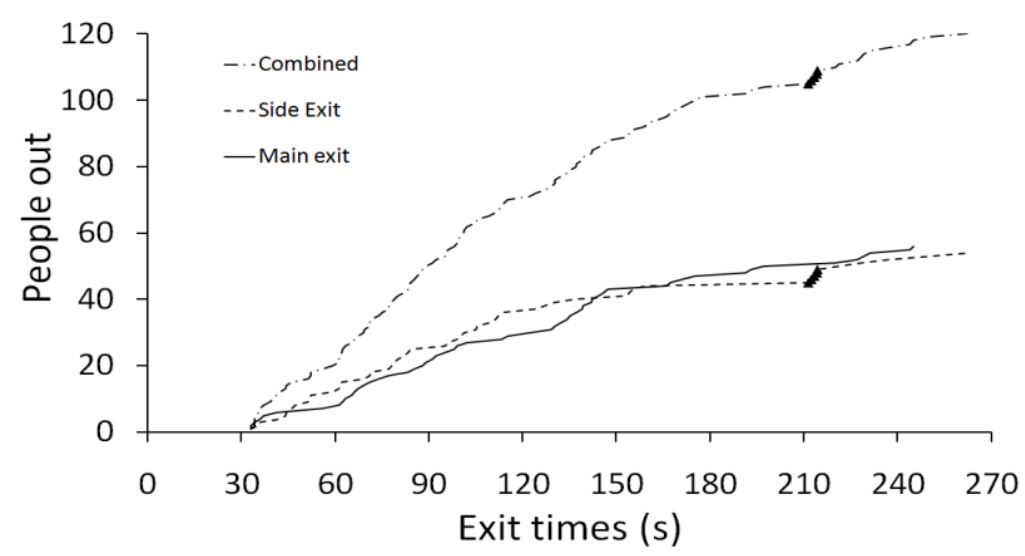

Fig. 3. Exit times measured in the Turkish evacuation trial.

Unfortunately, these curves are not very useful for model validation for several reasons. Firstly, they include data from library staff and cleaners. The difficulty with including this data is that the response times for these occupants could not be determined from the video analysis. This is primarily because these occupants were not in the monitored regions. It is inappropriate to assume that the response times for these occupants can be derived from the student response time distribution as the response for staff can be expected to be different to that of students. In addition, the starting locations of these staff are also unknown as they were not initially located in any of the monitored locations. Finally, once staff have responded to the alarm, they can be expected to have tasks to perform other than simply evacuate. Secondly, several students displayed atypical behavior which is not expected to be predicted by evacuation models. First, a student who passed through the exit turnstile beside the entry reception desk decided not to exit the building but remained at the reception desk and engaged in several discussions with other students and the library staff. She eventually decided to exit the library some time after having passed through the turnstile. Another group of five students (indicated by the triangles in Fig. 3) also displayed atypical behavior. This group of students approached the side exit, and while they could not be visually observed by the exit camera, their voices could be heard on the sound track. They appeared in the vicinity of the side exit location approximately $159 \mathrm{~s}$ after the start of the alarm and started talking amongst themselves and also with the staff member who was standing at the side exit. This discussion went on for approximately $50 \mathrm{~s}$ after which they decided to continue their journey and leave the building via the side exit. Due to the issues identified, these 14 staff and 6 students were removed from the exiting information. Prior to removing these staff and students from the exit count, 56 people used the side exit and 64 people used the main exit. After removing the identified staff and students the total number of students using the side exit was 44 while the total number of students using the main exit was 56 resulting in 100 students exiting the building. The time for the last person to use the side exit has decreased from $262.1 \mathrm{sec}$ to $159.1 \mathrm{~s}$ due to removing the staff and atypical students from this exit. There has been no change in the times for the main exit. When the exit usage for both exits is combined, the time for the last person to exit the building is reduced from $262.1 \mathrm{~s}$ to $245.2 \mathrm{~s}$. The modified exit curves are shown in Fig. 6a and are considered appropriate for model validation purposes.

To complete the validation data-set it is necessary to specify the starting locations for the 100 students. The approximate starting location of 86 students was determined from video footage information. These people were located within the six monitored zones of the library. The locations of each of these zones are identified in Fig. 4 and the number of people within each of these zones is identified in Table 1. Unfortunately, the location of 14 students could not be determined. However, it is clear that these students did not originate from any of the six monitored zones. The location of these students must have been restricted to a small part of the library, which was open to students and not monitored. This region is identified in Fig. 4 as the hatched region. Furthermore, by knowing which exit people used it is possible to reasonably reduce this region down to that hatched using the diagonal stripes. 


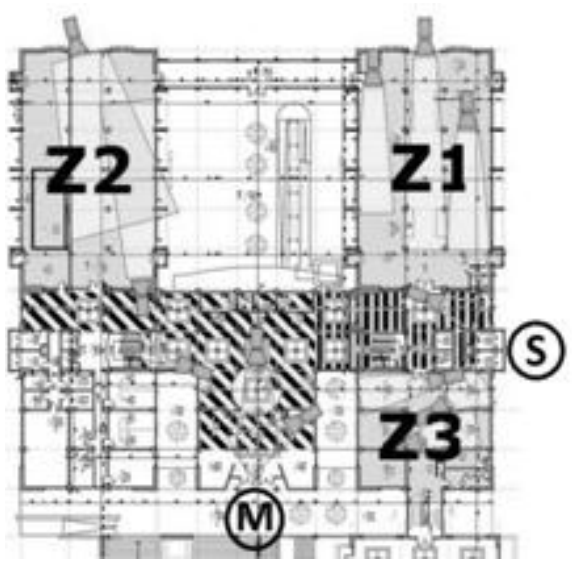

(a)

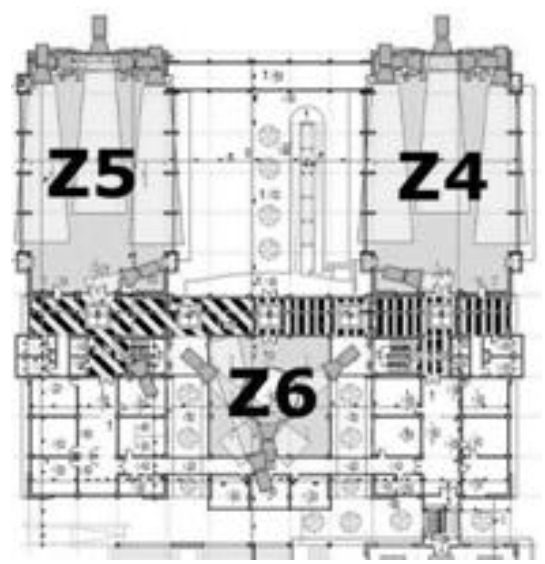

(b)

Fig. 4. Population distribution zones (M - Main exit, $\mathrm{S}$ - Side exit): (a) ground floor; (b) first floor.

Table 1. Number of students in each zone.

\begin{tabular}{|l|c|c|c|}
\hline Zone & $\begin{array}{c}\text { No. of people } \\
\text { with known } \\
\text { response times }\end{array}$ & $\begin{array}{c}\text { No. of people with } \\
\text { unknown } \\
\text { response times }\end{array}$ & $\begin{array}{c}\text { Total } \\
\text { number of } \\
\text { people }\end{array}$ \\
\hline Z1 & 6 & 5 & 11 \\
\hline Z2 & 9 & 14 & 23 \\
\hline Z3 & 20 & 2 & 22 \\
\hline Z4 & 4 & 10 & 14 \\
\hline Z5 & 7 & 3 & 10 \\
\hline Z6 & 5 & 1 & 6 \\
\hline Unknown & \multicolumn{3}{|c|}{} \\
\hline
\end{tabular}

Table 2. Response time data for each zone.

\begin{tabular}{|l|c|c|c|r|}
\hline Zone & $\begin{array}{c}\text { Mean of } \\
\text { logged } \\
\text { response } \\
\text { times }\end{array}$ & $\begin{array}{c}\text { Std dev. } \\
\text { of logged } \\
\text { response } \\
\text { times }\end{array}$ & $\begin{array}{c}\text { Minimum } \\
\text { measured } \\
\text { response time } \\
(\mathbf{s})\end{array}$ & $\begin{array}{c}\text { Maximum } \\
\text { measured } \\
\text { response time } \\
(\mathbf{s})\end{array}$ \\
\hline $\mathrm{Z1}$ & 3.94 & 0.71 & 19.24 & 108.24 \\
\hline $\mathrm{Z} 2$ & 3.56 & 0.46 & 22.02 & 72.17 \\
\hline $\mathrm{Z3}$ & 3.75 & 0.72 & 11.00 & 116.03 \\
\hline $\mathrm{Z} 4$ & 3.70 & 0.47 & 22.01 & 184.08 \\
\hline $\mathrm{Z5}$ & 4.44 & 0.85 & 25.07 & 151.17 \\
\hline $\mathrm{Z6}$ & 4.23 & 0.92 & 16.00 & 181.04 \\
\hline Remaining areas & 3.78 & 0.72 & 11.00 & \\
\hline
\end{tabular}

The remaining parameter that must be determined is the response time distribution. There are two ways in which the response time can be allocated to the building occupants. In the first approach individual response times based on the log-normal curve derived from the global response time distribution presented in Fig. 2 are imposed on the population. This means that occupants would be assigned a response time based on the global distribution, irrespective of which region they were initially located in. Alternatively, a log-normal response time curve for each zone can be produced, based on the measured response time data derived from each zone. Table 1 shows the number of people in each zone for which response time data is 
available. Using this approach, those people who are known to have originated from a particular zone, but for which a response time could not be determined will assume the response time distribution associated with that zone. Presented in Table 2 is the range of response times measured for each zone along with the parameters for the associated log-normal curve. Also shown in Table 2 are the response time parameters for the occupants that could not be assigned to one of the zones. The response time distributions for each zone based on the data presented in Table 2 is presented in Fig. 5.
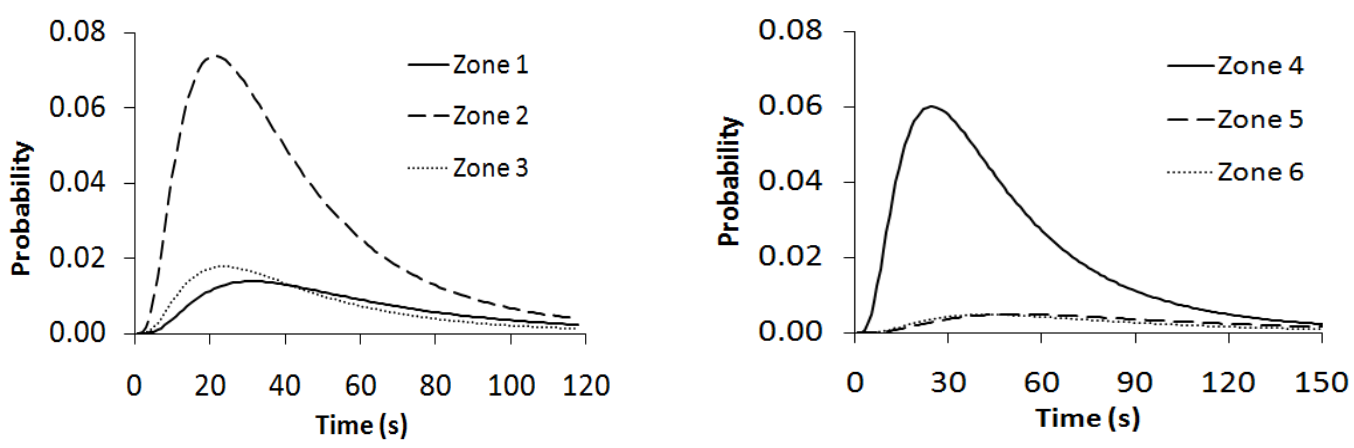

Fig. 5. Response time distribution for individual zones.

\section{MODELLING RESULTS AND DISCUSSION}

The buildingEXODUS software was used to undertake some preliminary simulations of the Turkish library evacuation and compare these predictions with the TEVDS. The buildingEXODUS software has been described many times in the literature [8] and so is not discussed again here. The geometry of the structure was constructed using CAD drawings of the building. The population was distributed according to Table 1, with the people from unknown areas distributed as follows, two people on the upper floor and 12 people on the lower floor in the diagonal hatched areas shown in Fig. 4. Only two of the 14 unknown people were placed on the upper floor as it is known that only 12 people used the staircase located opposite Zone 5 and there were 10 people initially located in Zone 5. Furthermore, it is known that 20 people used the staircase located opposite Zone 4 and there are 20 people located in Zones 4 and 6 . The response times for the agents in the model was specified using the second of the two approaches identified above as this is considered to be the most accurate approach. Due to space limitations, it is left for further work to repeat this analysis using the global response time distribution. Thus the response time distributions specified in Table 2 was used to provide response times for each of the agents in each of the zones. The remainder of the agent parameters was specified using the buildingEXODUS default parameters.

Using this set-up, the initial set of simulations were run so that the agents would utilize their nearest exits. With this configuration, approximately 47 agents made use of the main exit and 53 agents made use of the side exit. These results are counter to expectations as more agents are utilizing the side exit than the main exit and we know that the side exit has NEVER been previously used by students. It is more likely that the majority of people would make use of the main exit as it is the exit that the students use on a normal basis. This is indeed what happened in the experiment, 56 students used the main exit and 44 students used the side exit. On the ground floor, the students in Zones 1 and 3 exit their zones and enter the cross-corridor immediately adjacent to the side exit. It is unlikely that any of these students would elect to re-direct to the main exit as they are immediately adjacent to the side exit. Furthermore, a staff member stood by this exit for most of the evacuation, and encouraged the students to use the side exit. Thus, nine of the 20 students from the upper floor who use the staircase opposite Zone 4 must elect to use the main exit rather than the side exit. As they exit from the stair they are closer to the side exit than the main exit however, their back is directed towards the side exit, they face the direction of the main exit and they normally make use of the main exit. It is thus reasonable to assume that the required nine students who use this stair elect to use the main exit rather than the side exit. There is also some evidence to support this assumption from the video footage data.

A second preliminary scenario was set up in which the agents were again directed to their nearest exits, but in additional there was a $45 \%$ probability that agents descending from the staircase opposite Zone 4 would 
redirect towards the main exit. The results for this scenario are presented in Fig. 6. From Fig. 6 it is clear that the software produces a reasonable representation of the overall evacuation, with the broad trends in evacuation performance being captured by the simulation. However, the predicted evacuation performance is somewhat faster than the actual evacuation performance. A total of 20 repeat simulations were run in which only the initial positions of the 14 unknown people was randomized within the designated area. Fig. $6 \mathrm{~b}$ shows the overall comparison between predicted and measured egress curves for the total number of people exiting the building. It is noted from Fig. $6 \mathrm{~b}$ that there is little difference between the predicted maximum and minimum curves. As can be seen from Fig. $6 \mathrm{~b}$, the overall agreement between predicted and measured egress times is quite good over the first $35 \%$ of the evacuation, as measured by the number of people who have successfully evacuated. However, the predicted curve deviates from the measured results over the next $60 \%$ of the evacuation, with the predicted egress times being considerably faster than those observed in the actual evacuation. During this period the largest difference in predicted egress times occurs when $92 \%$ of the population has evacuated. The predicted time for the evacuation of 92 occupants is 144.9 $\mathrm{s}$ while the measured time is $191.2 \mathrm{~s}$, an under-prediction of $46 \mathrm{~s}$ or $24 \%$. The largest difference in the number of people who have successfully evacuated occurs at approximately $120 \mathrm{~s}$. At this time, the predicted average number of people who have evacuated is 83 while the actual number that has evacuated is 66 , an over-prediction of $26 \%$. Over the final $5 \%$ of the evacuation, the differences between the predicted and measured results decrease. The final average predicted evacuation time is $232.0 \mathrm{~s}$ compared with the measured value of $245.2 \mathrm{~s}$, an under-prediction of only $5.4 \%$.

The exit curve for the side exit is presented in Fig. 6c. It is noted that while the predicted maximum and minimum curves are similar for most of the evacuation, they differ significantly for the last four agents. The minimum predicted evacuation time is $137.8 \mathrm{~s}$ while the maximum predicted evacuation time is $232.1 \mathrm{~s}$ with an average evacuation time of $216.6 \mathrm{~s}$. The evacuation times for the last four agents is highlighted on each curve in Fig. 6c. As can be seen, there is considerable variation between the time for the last two agents in each of the simulations. This is the result of the probabilistic selection of the nine agents using the staircase that are directed to use the main exit. In the cases which produce the shortest egress times, some of the randomly selected nine agents may have had long response times or have been located in the remotest part of the affected zones and thus have had long travel times or both. If these agents are diverted to the main exit their egress times will not contribute to that of the side exit. However, if they are not diverted to the main exit they will utilize the side exit and thus their long egress times will contribute to that of the side exit. This will lead to some of the simulations having long total egress times and hence will push the average total egress time up for this exit. However, it is noted that there is very good agreement between the predicted and measured use of the side exit over the first $91 \%$ ( 40 people) of the evacuation, as measured by the number of people who have successfully evacuated. At $100 \mathrm{~s}, 29$ people were observed to have exited using the side exit, whereas the predicted number varies from 30 to 34 with an average of 32. Indeed, we note that over much of this period, the measured curve falls between the predicted minimum and maximum curves. The difference between the predicted and measured egress times begins to increase after this point. The measured evacuation time for 41 people ( $93 \%$ of the population) to exit the geometry through the side exit is $153.2 \mathrm{~s}$ while the predicted minimum, average and maximum times are; $122.0 \mathrm{~s}$ (20\% under-predicted), $133.5 \mathrm{~s}$ (13\% under-predicted) and $136.9 \mathrm{~s}$ (11\% underpredicted) respectively. However, the total measured evacuation time for the side exit is $159.1 \mathrm{~s}$ while the predicted minimum, average and maximum times are; $137.8 \mathrm{~s} \mathrm{(13 \%} \mathrm{under-predicted),} 216.6 \mathrm{~s} \mathrm{(36 \%} \mathrm{over-}$ predicted) and $232.1 \mathrm{~s}$ (46\% over-predicted) respectively. As already highlighted, this large difference is due to the random allocation of the last two agents to the side exit. The exit curve for the main exit is presented in Fig. 6d. We note that there is little difference between the predicted maximum and minimum curves. As can be seen from Fig. 6d, the overall agreement between predicted and measured egress times is quite good over the first 30\% (17 people) of the evacuation, as measured by the number of people who have successfully evacuated. However, the predicted curve deviates from the measured results over the next $60 \%$ of the evacuation, with the predicted egress times being considerably faster than those observed in the actual evacuation. In this respect, the curve for the main exit closely follows the trends found in the overall exit curve presented in Fig. 6b. By the time that 20 people have evacuated ( $36 \%$ of the population), the average predicted egress time under-predicts the actual egress time by 13\% (11.4 s) and this increases to an under-prediction of $33 \%$ ( $63.5 \mathrm{~s}$ ) by the time 48 people have evacuated ( $86 \%$ of the population). However, average predicted total evacuation time for the main exit is $229.2 \mathrm{~s}$ while the measured total evacuation time is $245.2 \mathrm{~s}$, an under-prediction of only $6.5 \%$. 


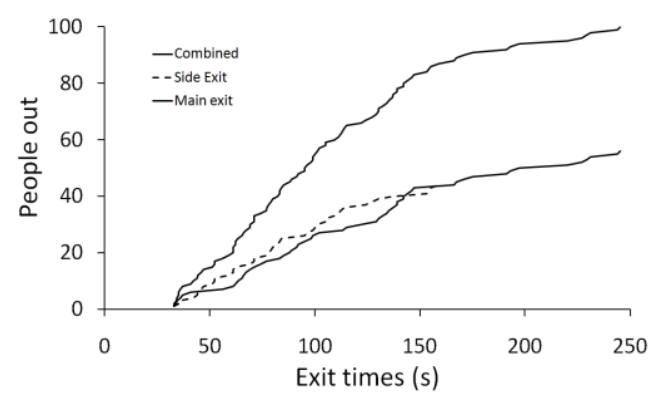

(a)

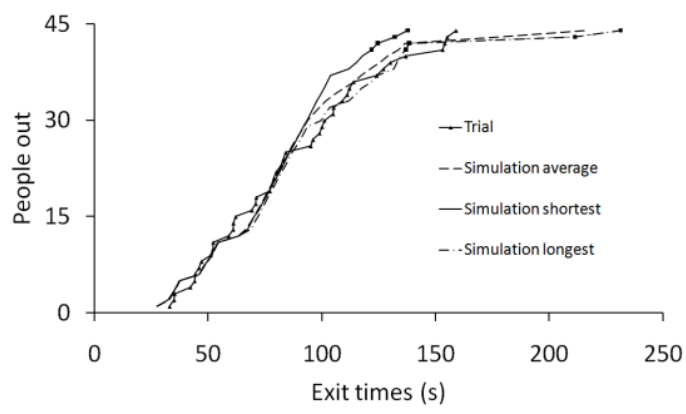

(c)

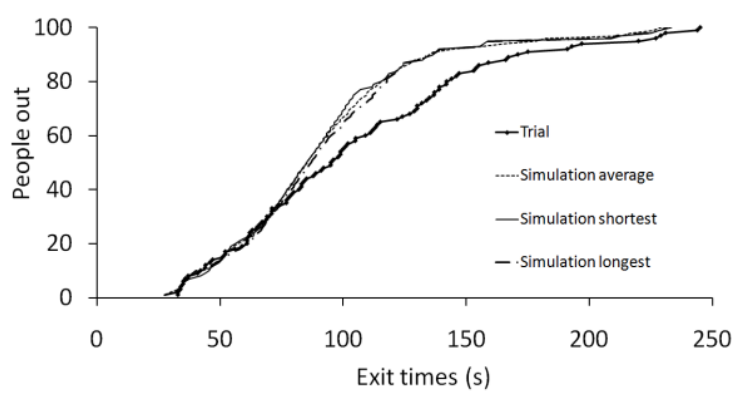

(b)

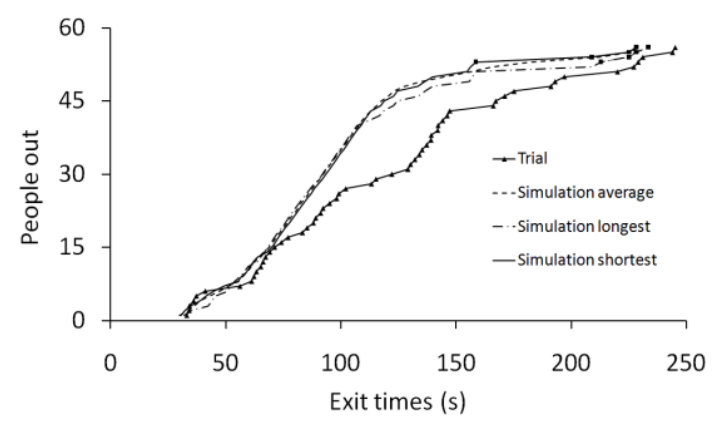

(d)

Fig. 6. Measured and predicted exit graphs: (a) modified exit times measured in the Turkish evacuation trial; (b) total number of people out of the building as a function of time; (c) number of people out of the side exit as a function of time; (d) number of people out of the main exit as a function of time.

Clearly there are significant differences between the predictions of the evacuation through the main exit and the measured values and this impacts the overall level of agreement between the predicted and measured evacuation. Closer examination of the video footage revealed two possible contributory causes for these differences. First, the population appeared to move relatively slowly during the entire evacuation. This was also noted by observers during the actual evacuation. The students did not appear to move with any degree of urgency. Within buildingEXODUS the default 'Fast Walk' travel speeds are distributed between $0.81 \mathrm{~m} / \mathrm{s}$ and $1.5 \mathrm{~m} / \mathrm{s}$ and the average 'Fast Walk' travel speed for the population was $1.3 \mathrm{~m} / \mathrm{s}$. From the video footage it was possible to measure the travel speeds of 16 people ( $16 \%$ of the population), these ranged from $0.6 \mathrm{~m} / \mathrm{s}$ to $1.3 \mathrm{~m} / \mathrm{s}$ with an average of $1.0 \mathrm{~m} / \mathrm{s}$. These walking speeds were measured for individuals who were not travelling with large groups of people or moving through congested spaces. They were measured for essentially free walking individuals. From this sample it appears that the default travel speed distribution used by buildingEXODUS results in travel speeds which are on average $30 \%$ higher than that observed. Clearly this will have an impact on the overall evacuation results. However perhaps of more importance is the exiting behavior through the main exit. It was noted from the video footage that crowding did not develop by the main exit but was observed to occur before the exit, at the entry of the turnstile. At times, the turnstile proved to be a significant bottleneck as students struggled to find their ID cards - even though ID cards were not required. Assuming an average travel speed of $1.0 \mathrm{~m} / \mathrm{s}$ and an unhindered flow, it should take approximately $1.0 \mathrm{~s}$ for each person to pass through the $1.0 \mathrm{~m}$ wide turnstile. Measurements of the passage time through the turnstile vary from a minimum of $1.2 \mathrm{~s}$ to a maximum of $7.9 \mathrm{~s}$, with an average of $2.7 \mathrm{~s}$. As the population is delayed in passing through the turnstile they are delayed in reaching and passing through the main exit. Several of the plateaus in exit-time graph for the main exit (Fig. 6d) correspond to the times of significant delay through the turnstile.

The computer simulations presented in this paper did not include a representation of the turnstile or the lower than expected average travel speed of the population. The model is being modified to represent the movement of people through the turnstile and to represent the lower travel speeds of the population to investigate the impact that these may have on the simulated evacuation times. 


\section{CONCLUSIONS}

A framework to enable the systematic analysis of Response Phase behaviors has been developed and applied to an unannounced evacuation trial in a university library in Turkey. The framework not only provides a consistent method for describing Response Phase behavior, but also provides a framework for classifying and quantifying the Response Phase other than simply using the overall response time. By understanding and quantifying the factors which influence and ultimately determine the Response Phase we are better able to compare and contrast different evacuation situations. In addition, the framework provides an empirical means of predicting population average response times based on average number of Information/Action tasks, average task duration and average notification time.

The Turkish evacuation produced a response time distribution which follows a log-normal curve as is commonly found in evacuation trials. The average response time extracted from the 51 analyzed people was $56.1 \mathrm{~s}$, and ranged from $11 \mathrm{~s}$ to $181 \mathrm{~s}$. Using the framework the Response Phase behaviors were analyzed and decomposed into their constituent components. The results of this analysis suggests the following; the average duration of the Notification stage was $10.0 \mathrm{~s}$, the average number of Action Tasks undertaken was 3.5, the average number of Information Tasks was 5.1, the average duration of an Action Task was $5.2 \mathrm{~s}$, and the average duration of an Information task was $4.7 \mathrm{~s}$. This basic information provides a means of characterizing the Response Phase of the Turkish library evacuation which goes far beyond simply measuring the response time. A similar analysis has already been undertaken for an evacuation in a library in the Czech Republic. Two further analyses are currently underway for two other library evacuations, one conducted in Poland and one in the UK. A key aim of this work is to investigate whether culture plays a significant role in evacuation behavior. Using the framework, it is planned to explore whether social culture will impact behavioral determinates such as number, type and average duration of tasks performed in the Activity stage and the duration of the Notification stage. It should be noted that even if these factors are not dependent on social culture, they are expected to be dependent on the nature of the environment e.g. library, shopping complex, high-rise building, the nature of the notification system e.g. staff intervention, alarm, voice alarm, and the level of training of the occupants. Thus, it will be important to determine the dependence of the identified behavioral determinates on type of environment, type of notification system and degree of training.

As part of this work an empirical response time model has been developed based on the analysis of Response Phase behaviors. This model was previously applied to the Czech Republic data-set and found to produce an accurate prediction of the average response time for that evacuation. In the work presented in this paper, the same model (using the same constants) was applied to the Turkey data-set and was also found to produce a good prediction for the average response time. Thus the constants used in the empirical response time model appear to be reasonably robust, providing a good level of agreement for two evacuation trials conducted in two different libraries in two different countries. The empirical response time model will also be applied to data derived from two additional evacuation trials, one in Poland and one in the UK. If the empirical response time model can be demonstrated to be robust, its value goes beyond simply being a means of determining average response times for a population. Of more importance is the insight it provides into the behavioral factors driving the response time. Using this approach it should be possible to estimate the impact of introducing technical or procedural measures to address various behavioral determinants of response time such as the duration of the average Information Task, or the number of Information Tasks that are performed by a population.

Data from the evacuation trial was also collected to produce an evacuation model validation data-set. This data consisted of the population starting locations, a global response time distribution for the population, local response time distributions for sub-populations, exit usage, exit time curves for each exit and the overall exit time curve. While the total data-set is available, the data-set was also cleaned to remove six students with atypical exiting behaviors (from the exit data only) and 14 staff (from both the response time data and the exiting data), for which little information is available. Preliminary simulations using the buildingEXODUS software revealed that several differences between model predictions and measured results exist. These are thought to be due to two factors, the behavior of students as they passed through a turnstile prior to one of the final exits - which was not modeled, and the movement speed of the student population, which is on average $30 \%$ lower than the default average walk speed used in the software. These factors will be investigated in further modeling work associated with this project along with the nature of the response time distribution (global or local). Finally, it is worth noting that the nature of the fire alarm 
training regime, the nature of the alarm testing regime and the nature of the voice alarm messages and the status of the emergency exits are all examples of how local fire culture can impact overall evacuation performance.

\section{ACKNOWLEDGEMENTS}

Project $\mathrm{BeSeCu}$ (contract 218324) is funded under the European Union Framework 7 Security initiative. The authors acknowledge the co-operation of their project partners. The authors are also indebted to Izmir Yuksek Teknoloji Enstitusu for allowing the project team to make use of their library building, to their students for their co-operation and to Mr Gultekin Gurdal, the library Director and all his staff for their assistance, without whom this work would not have been possible.

\section{REFERENCES}

[1] Galea, E.R., Deere, S., Sharp, G., Filippidis L., and Hulse, L., "Investigating the impact of culture on evacuation behaviour," Proceedings of the 12th International Conference, Interflam 2010, Intersciences, 2010, pp. 879-892.

[2] Galea, E.R., "Evacuation Response Phase Behavior," CMS Press 09/IM/147, London, 2009, pp. 130.

[3] Sime, J., "Human Behavior In Fires Summary Report," CFBAC Rept No.450, Portsmouth, 1992, pp. 1-20.

[4] Sime, J., "Escape Behavior In Fires And Evacuations," Fires And Human Behavior, Fulton, London, 1990, pp. 56-87.

[5] Proulx, G., "Time Delay To Start Evacuating Upon Hearing The Fire Alarm," Human Factors And Ergonomics Society 38th Annual Meeting, Ergonomics Society, 1994, pp. 811-815.

[6] Proulx, G. and Sime, J., (1991) To Prevent 'Panic' In An Underground Emergency: Why Not Tell People The Truth?, Fire Safety Science 3: 843-852, http://dx.doi.org/10.3801/IAFSS.FSS.3-843.

[7] Galea, E.R., Hulse, L., Day, R., Siddiqui, and A., and Sharp, G. , "The UK WTC9/11 Evacuation Study: An Overview of the Methodologies Employed and some analysis relating to fatigue, stair travel speeds and occupant response times," Human Behavior in Fire, 4th Int. Symp, Intersciences, 2009, pp. 27-40.

[8] Galea, E., Wang, Z., Veeraswamy, A., Jia, F., Lawrence, P., and Ewer, J., (2009) Coupled Fire/Evacuation Analysis of the Station Nightclub Fire, Fire Safety Science 9: 465-476, http://dx.doi.org/10.3801/IAFSS.FSS.9-465. 